phical system of the Middle Ages as it is formulated, for example, in the writing of Thomas Aquinas. For the same philosophies from which the geocentric system in astronomy could be derived, one could also derive the mediaeval ideas about religion, morals, and politics on which that "good conduct of man" was based. The choice of the heliocentric system would have disappointed this philosophy and had, according to the opinion of its advocates, led to disastrous changes in human behaviour, religious as well as political. We can easily observe that the same way of metaphysical and sociological interpretation has occurred in every period of history, whenever general theories could not be satisfactorily validated by purely scientific methods. We may exemplify this situation by theories of the 19th and 20th centuries if we consider sweeping hypotheses like "there is no ether", "there is no evolution from monkey to man", "there is no mechanical explanation for human behaviour", etc. In all these cases, we easily observe the tendency to investigate directly the plausibility of these hypotheses from the viewpoint of common sense. But we can also easily observe the influence of human behaviour which is ascribed by powerful groups to these hypotheses.

\title{
Mind-like Behaviour in Artefacts
}

\author{
BY DR. D. M. MACKAY.
}

\section{ABSTRACT of Paper read on 13th November, 1950.}

This paper is not concerned with analogies between contemporary computing machines and brains, nor with much that has found itself entitled "Cybernetics". Its purpose is firstly to examine the extent to which in principle an artificial organism could parallel human activity, particularly those aspects by which we "justify the inference to other minds", and secondly to indicate some of the philosophical issues to which the possibilities discussed are relevant.

Familiar faculties of artificial goal-seeking mechanisms are briefly described, by way of introduction to a probabilistic reasoning-mechanism which, it is suggested, might in principle parallel all describable forms of human behaviour. For this mechanism, the meaning of a receptum is represented by a probabilityspectrum over a set of possible responses by the mechanism. These responses may be internal, directed to the alteration of an internal formal representation of "that which is the case". This activity, it is suggested, distinguishes recognition from reception, in that the act of response by (formal) replication entails symbolic activity equivalent to the naming of the receptum.

The problem of abstraction is that of the naming of an invariant in the flux of recepta. It is suggested that if the elementary concepts of the universe of discourse are (and are symbolized or named by arousal of) the elementary component-acts of response, then the possession of an invariant mode of equilibrant response to a specific invariant, enables the artefact ipso facto to name the invariant under all its transformations. It is even possible for the artefact to discover and name for itself new invariants, by a self-guiding statistical discovery-process, and to generate new hypotheses in the form of abstractions from abstractive activity. Analogues of emotional behaviour, the weighing of evidence-prejudiced or otherwise-and other characteristically human activity suggest themselves automatically in terms of this probabilistic mechanism ; words such as personality, consciousness, and self-consciousness seem to admit of consistent interpretation in such terms. Choices can be statistically reasonable yet individually unpredictable in principle, 
These possibilities bear on several lines of thought. Mindlike behaviour in the artefact is seen to be characterized by the circulation of information; and any mode of analysis which makes the latter concept meaningless will exorcise the concept of mind with Procrustean thoroughness. It is suggested that an analogue of the Principle of Complementarity admits of important application here, and that criteria of appropriateness as well as of consistency must be used to test the legitimacy of an analysis.

The artefact is not advanced as a detailed model of the human brain; but the manner in which mentality could be mediated by it, and the nature of its choices, are suggestive. It offers an interpretation of the " $I$ " as the totality of currently-reactive elements organized by information-linkages to constitute an organism ; and the concept of "randomness" here appears in a somewhat new light as the element responsible for the interest and creativeness possessed by the artificial personality so mediated. The analogue of human free will is seen to have a natural place within the framework of physical "law" governing the mechanism.

It is finally suggested that no new theological issues are raised by such developments, though misconceptions of Christian doctrine, both by some Christians and by their opponents, make it important that the " nature of Man " be carefully studied in this new light. To build an artificial man is doubtless impracticable; but the possibility in any case affects in no way the status of choices made by natural men in terms of the calculus of responsibility.

\section{The Problem of the Existence of Mathematical Entities}

\section{By Profrssor E. W. BETH}

\section{ABSTRACT of Paper read on 2nd March, 1951.}

The problem of the existence of mathematical entities takes its origin from the fact that, while the truths of mathematics belong to those elements in human knowledge to which we ascribe the highest degree of certainty, we search in vain, in the world of human experience, for entities which present the properties described in these truths. The first attempts to solve it: platonism, aristotelianism, and constructivism (Plotinus, Nicolaus Cusanus, Kepler, Hobbes), belong to speculative philosophy.

The crisis in the foundations of mathematics, however, forced the mathematicians to consider the problem from their own point of view. At first, they borrowed their solutions from speculative philosophy. Later, the development of axiomatics in geometry led Poincaré and Hilbert to accept the formal consistency of a system of axioms as a necessary and sufficient condition for the existence of a model. It was observed by L. Löwenheim (1915) that, for axiom systems formulated within the Peirce-Schröder calculus, the existence of a model can be discussed in a rigorous manner. The development of his ideas led finally to the Löwenheim-Skolem-Gödel theorem : an axiom system formulated within elementary logic has a model, if and only if it is formally consistent. Recently, this result has been extended to various other logical systems by A. Mostowski, L. Henkin, and Helena Rasiowa.

Far from leading to a final settlement of the problem, however, this result was shown by Th. Skolem to give rise to a complete relativisation of mathematical existence. So it seems reasonable to reconsider the problem from the point of view of speculative philosophy ; of course, we cannot expect to obtain, on this basis, a deductive theory of mathematical existence. Nevertheless, it is felt that a realistic and pluralistic conception of human knowledge, which can borrow precious elements from older doctrines, may contribute to a clarification of the situation. 